\title{
Achados clínicos da sensibilidade a analgésicos e antiinflamatórios não-hormonais
}

\author{
N.A. RosÁrio, A.C. Ribeiro \\ Hospital de Clínicas da Universidade Federal do Paraná, Curitiba, PR
}

RESUMO - Analgésicos (ANA) e antiinflamatórios não-hormonais (AINH) podem causar reações que simulam as alérgi cas ou agravam asma e urticária.

Овј етіvo. Verificar as manifestações clínicas de pacientes com história de reação a analgésicos (ANA) e antiinflamatórios não-hormonais (AINH).

Método. Análise retrospectiva de prontuários de 183 pacientes com história de sensibilidade a ANA e AINH.

Resultados. Eram 93 (51\%) pacientes do sexo feminino e 90 (49\%) do sexo masculino; 63 (34\%) com idade igual ou inferior a 15 anos e 120 (66\%) com idade superior a 15 anos. Havia um predomínio de pacientes do sexo feminino com idade superior a 15 anos que foi estatisticamente significativo $(p=0,02)$. A idade por ocasião da primeira reação com medicamentos variou de 7 meses a 65 anos (média de 15 anos). Testes cutâneos para aeroalérgenos foram positivos para pelo menos um alérgeno testado em 100/138 (72\%). As manifestações clínicas encontradas foram angioedema (86\%), urticária (39\%), reações sistêmicas

\section{NTRODUÇÃO}

Há 100 anos o ácido acetilsalicílico (AAS) tem sido utilizado por bilhões de pessoas como o primeiro antiinflamatório não-hormonal (AINH), bem como analgésico (ANA) e antipiréticoํ. A partir de 1960, uma série de outros AINH foram sintetizados e administrados não só para doenças musculoesquel éticas, mas para inflamação em geral e cólica menstrual.

A cicloxigenase, enzima responsável pela geração de prostaglandinas (PG) a partir do ácido araquidônico se apresenta sob uma forma induzida (COX-2) e uma isoforma diferente, constitutiva (COX-1). A COX-2 é indetectável nos tecidos em condições fisiológicas, mas aumenta sua expressão em até 80 vezes durante inflamação ou estímulo mitogênico².

O estímulo à COX-1 regula processos fisiológicos normais e é responsável pela síntese de prostaglandinas $^{3,4}$. O mai or benefício dos inibidores seletivos de
(30\%), reações nasais e oculares (15\%) e crise de asma (14\%). Não havia diferença quanto à freqüência de sintomas com relação à idade. Havia história familiar de sensibilidade a ANA/AI NH em sete pacientes $(3,8 \%)$. As doenças associadas foram rinite (55\%), urticária crônica (47\%), asma (37\%) e conjuntivite (17,5\%). As drogas causavam crise de asma com maior freqüência em asmáticos do que em não asmáticos $(p=0,001)$. Reações repetidas a mais de uma droga ocorreram em 107 (58\%) pacientes.

Conclusões. Reações a ANA e AINH foram freqüentes em atópicos; crianças e adultos reagiam igualmente; foram mais comuns em adultos do sexo feminino; angioedema palpebral foi a manifestação clínica mais freqüente; broncoespasmo foi mais comum nos asmáticos e a maioria dos pacientes tinha reações repetidas a mais de uma droga.

UnITERmos: Antiinflamatório. Alergia. Asma. Ácido acetil salicílico.

COX-2 é um perfil favorável em efeitos colaterais, sem a perda da atividade antiinflamatória².

A exposição ao AAS e aos AINH é bem tolerada pela maioria dos indivíduos. Alguns desenvolvem efeitos adversos que incluem reações alérgicas e pseudoalérgicas. Reações cruzadas respiratórias (rinoconjuntival e asma) e reações cruzadas cutâneas (urticária/angioedema) são ligadas ao efeito comum de inibição da cicloxigenase pelo AAS e AI NH. As outras reações são específicas para cada droga e independentes do efeito de inibição da COX, incluindo urticária/angioedema ou anafilaxia, meningite asséptica e pneumonite de hipersensibilidade secundária. Estas reações são causadas por AAS ou um AINH específico ${ }^{5}$.

A inibição da COX por estes agentes parece ser responsável pelo desequilíbrio dos metabólitos do áci do araquidônico, com um aumento dos produtos derivados da lipoxigenase, os leucotrienos (LT), com propriedades broncoconstritoras e proinflamatórias ${ }^{6-9}$. 


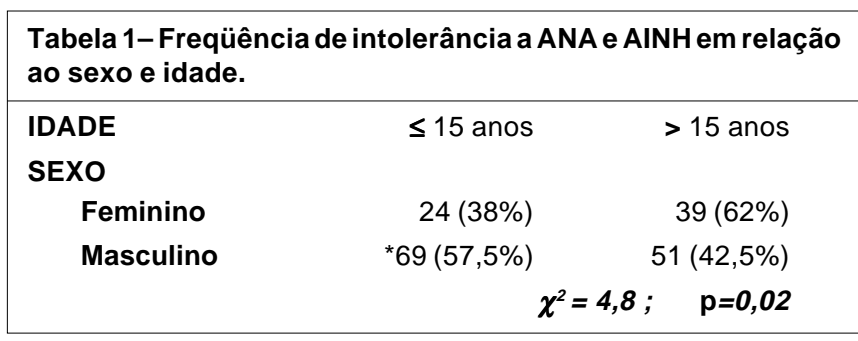

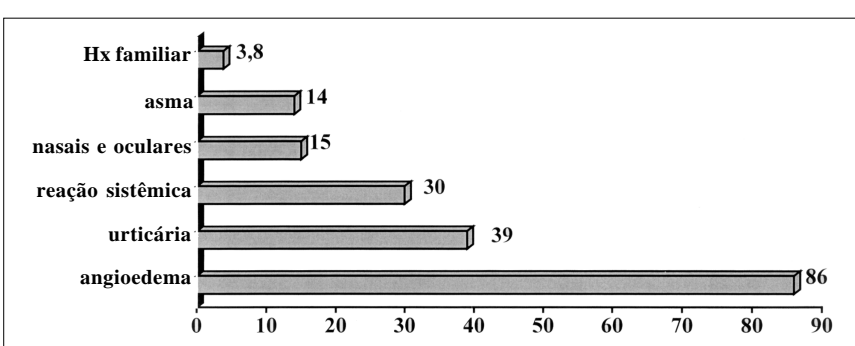

Fig. 1 - Manifestações clínicas mais freqüentes na sensibilidade a ANA e AINH $(n=183)$

A incidência de doenças atópicas em pacientes com sensibilidade a AINH não é conhecida. Para alguns autores há um aumento de sensibilidade a AINH em indivíduos atópicos, mas esta associação parece inexplicada ${ }^{10,11}$.

O objetivo do presente estudo foi verificar as manifestações clínicas em pacientes com história de reação a analgésicos (ANA)/antitérmicos e AINH, particularmente a relação com atopia.

\section{MÉTODOS E CASUÍSTICA}

O trabalho é descritivo e retrospectivo, baseado em revisão de prontuários médicos de 183 pacientes com história de reações à ingestão de anal gésicos (ANA)/antitérmicos e AINH, atendidos em clínica de I munologia entre abril de 1980 a dezembro de 1998. Eram 93 (51\%) do sexo feminino e 90 (49\%) do sexo masculino, 63 (34\%) com idade igual ou inferior a 15 anos e 120 (66\%) com idade superior a 15 anos. A idade, por ocasião da primeira reação com medicamentos, variou de 7 meses a 65 anos, com média de 15 anos. Cinqüenta e oito por cento dos pacientes tiveram reações repetidas a mais de uma droga em ocasiões diferentes.

Foram realizados testes cutâneos de leitura imediata pela técnica de puntura com extratos glicerinados de aeroalérgenos que incluíam Dermatophagoides pteronyssinus, epitélio de cão e gato, pólen de gramíneas, fungos do ar e baratas, todos obtidos de Greer Laboratories, Lenoir USA.

Os testes cutâneos foram considerados positivos quando a pápula formada tinha diâmetro igual ou maior que $3 \mathrm{~mm}$.

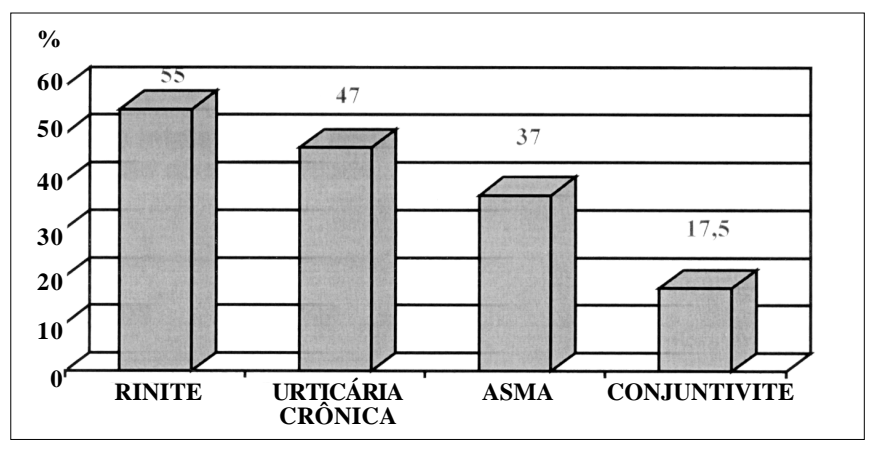

Fig. 2 - Doenças associadas em pacientes com sensibilidade a ANA e AINH. $(n=183)$

\section{RESULTADOS}

Com relação à idade e ao sexo, 24 (38\%) pacientes com idade inferior a 15 anos eram do sexo feminino enquanto que 69 (58\%) com idade superior a 15 anos eram do sexo feminino (tabela 1 ). 0 predomínio feminino nesta faixa etária era significativo $\left(\chi^{2}=4,8 ; p=0,02\right)$.

Os testes cutâneos com aeroalérgenos foram realizados em 138 pacientes, dos quais 100 (72\%) apresentaram resultado positivo, para pelo menos um alérgeno testado, e mais freqüentemente ao ácaro (92\%).

As manifestações clínicas encontradas foram angi oedema 158 (86\%), urticária 72 (39\%); reações sistêmicas 55 (30\%) ; reações nasais e oculares 28 (15\%) e crises de asma 24 (14\%). Havia história familiar de sensibilidade a AN A/AI NH em 7 (3,8\%) dos pacientes (fig.1). Quando a freqüência de manifestações clínicas foi analisada com relação à idade, verificou-se que não havia diferença significativa entre pacientes com idade inferior ou superior a 15 anos.

As doenças associadas à sensibilidade aos ANA e AI NH foram rinite 101 (55,2\%), urticária crônica 86 (47\%), asma 68 (37\%) e conjuntivite 32 (17,5\%) (fig. 2). Reações a uma droga isoladamente foram referidas ao ácido acetil salicílico em 55 (30\%); dipirona 14 (7,6\%); e AINH 8 (4,4\%). O acetaminofen, isoladamente, não desencadeou reações. Foram observadas reações repetidas a mais de uma droga em 106 (58\%) pacientes. O AAS e a dipirona estavam associados em 49 (26,7\%). Reações ao AAS e também a AINH ocorreram em 16 $(8,7 \%)$. I gual número de reações foram ocasionadas por AAS, dipirona e AINH. Apesar de o acetaminofen ser utilizado como al ternativa para casos de sensibilidade a outros antitérmicos/analgési cos, el e causou sintomas em $11 \%$ dos pacientes nas doses prescritas usualmente com esta finalidade, mas que não foram informadas (tabela 2).

Nossos pacientes não referiram se a nimesulida 


\begin{tabular}{|c|c|c|c|}
\hline $\begin{array}{l}\text { ISOLADAS } \\
(\mathrm{n}=77)\end{array}$ & & $\begin{array}{r}\text { MÚLTIPLA } \\
(n=106)\end{array}$ & \\
\hline \multirow[t]{2}{*}{ 1. AAS } & $55(30 \%)$ & $1+2$ & $49(26,7 \%)$ \\
\hline & & $1+3$ & $16(8,7 \%)$ \\
\hline 2. Dipirona & $14(7,6 \%)$ & $\begin{array}{l}1+2+3 \\
1+2+4\end{array}$ & $\begin{array}{r}16(8,7 \%) \\
8(4,4 \%)\end{array}$ \\
\hline \multirow[t]{2}{*}{ 3. $\mathrm{AINH}^{*}$} & $8(4,4 \%)$ & $2+3$ & $5(2,7 \%)$ \\
\hline & & $1+2+3+4$ & $4(2,2 \%)$ \\
\hline \multirow[t]{4}{*}{ 4. Acetaminofen } & 0 & $1+4$ & $4(2,2 \%)$ \\
\hline & & $2+4$ & $1(0,5 \%)$ \\
\hline & & $3+4$ & $2(1,0 \%)$ \\
\hline & & $1+3+4$ & $1(0,5 \%)$ \\
\hline
\end{tabular}

Tabela 3-Crises de broncoespasmo desencadeadas por drogas.

\begin{tabular}{|c|c|c|}
\hline Drogas & $\begin{array}{c}\text { Asmáticos } \\
(n=68)\end{array}$ & $\begin{array}{c}\text { Não-Asmáticos } \\
(n=115)\end{array}$ \\
\hline AAS & 9 & 3 \\
\hline AAS + Dipirona & 4 & 2 \\
\hline AAS + AINH & 2 & 0 \\
\hline AAS + Dipirona + AINH & 0 & 3 \\
\hline AAS + Dipirona + Acetaminofen & 0 & 1 \\
\hline Dipirona & 1 & 0 \\
\hline \multirow[t]{2}{*}{ Total } & $16(23,5 \%)$ & $9(7,8 \%)$ \\
\hline & $\chi^{2}=10,6$ & $p=0,001$ \\
\hline
\end{tabular}

havia sido utilizada ou não por eles. Os AINH que causaram reações foram propifenazona, ácido mefenâmico, diclofenaco, naproxeno, cetoprofeno e piroxicam. No grupo, havia 68 asmáticos e destes, $16(23,5 \%)$ apresentavam crise de asma com a utilização de medicamentos, na qual o AAS era responsável em 15 casos, dipirona em cinco casos, AINH em dois. Cento e quinze pacientes não eram asmáticos, porém nove apresentavam crises de broncoespasmo com a ingestão de AAS, seis tinham crises com a dipirona, três com AINH e um com acetaminofen (tabela 3 ).

A história de broncoespasmo à ingestão destas drogas era significativamente mais freqüente em asmáticos do que em não-asmáticos. $\left(\chi^{2}=10,6\right.$; $p=0,001$ ). Pólipos nasais foram encontrados à rinoscopia anterior em seis adultos, dos quais três eram asmáticos, porém cinco desenvolviam broncoespasmo à ingestão de AAS.

\section{DISCUSSÃO}

Os AINH têm em comum com o ácido acetil salicílico (AAS) os efeitos farmacológi cos pela inibição da ciclooxigenase $(\mathrm{COX})^{3}$. Estes efeitos compartilhados são essenciais para a eficácia como analgésicos e antiinflamatórios, mas também nos mecanismos de alguns efeitos colaterais destas drogas $^{1}$.

A principal causa de reação à droga é a ingestão de AAS e AINH. Um estudo retrospectivo de 266 indivíduos trazidos ao Serviço de E mergência, com reação anafilática, mostrou que em 52 casos (20\%) havia suspeita de reação à droga, dos quais 27 reagi ram à provocação com aspi rina e sete a AI NH . Portanto, cerca da metade dos casos com anafilaxia a drogas foi provocada por AAS e AINH ${ }^{12}$.

A prevalência de hipersensibilidade a AAS/ AINH é de $0,3 \%$ na população adulta e pediátrica. Está presente em cerca de $10 \%$ de adultos asmáticos e aumenta para 30 a $40 \%$ em pacientes com asma e polipose nasal ${ }^{5,13}$. Para Stevenson, autoridade reconhecida em reações ao AAS e AINH, o termo sensibilidade é preferível à intolerância, pois este último é menos específico e melhor descritivo para as reações gastro-intestinais causadas pel o $A_{A} S^{5,14}$.

Os mecanismos de sensibilidade à aspirina permanecem obscuros, mas há evidências de que as reações não sejam fenômenos de natureza imunológica ${ }^{15}$. A patogênese das doenças respiratórias provocadas por AAS envolve mastócitos e eosinófilos pela formação contínua de produtos do ácido araquidônico. Há uma correlação positiva entre a potência in vitro como inibidor da COX e a capacidade de induzir asma. A sensibilidade ao AAS resulta em tolerância cruzada a AINH estruturalmente não relacionados, mas com propriedades farmacológi cas similares. A inibição da COX poderia induzir sintomas por vários mecanismos: a) desequilíbrio na geração de prostaglandinas (PGs) e tromboxanos (TX) com ações protetoras; b) desvio do metabolismo do ácido araquidônico para a via da lipoxigenase, levando à maior produção de leucotrienos; c) ativação direta da lipoxigenase pelo $A A S^{5,15}$.

Ferreri et al. ${ }^{16}$ encontraram aumento de $\mathrm{LTC}_{4} \mathrm{e}$ histamina e diminuição de $\mathrm{PGE}_{2}$ em lavado nasal após provocação oral com AAS em asmáti cos sensíveis ao AAS. Achados semel hantes foram descritos por Kowalski et al ${ }^{15}$.

Sladek et al. ${ }^{17}$ obtiveram lavados broncoalveolares (LBA) 30 minutos após inalação de lisinaaspirina em asmáticos sensíveis ao AAS. Proteína alcalina principal, um mediador químico proinflamatório do eosinófilo, LTC $_{4}$ e histamina estavam aumentadas e $\mathrm{TXB}_{2}$ diminuída, em relação aos valores basais antes da provocação.

Portanto, duas células, os mastócitos e eosinófilos, são provavelmente responsáveis pelas reações ao AAS, através da síntese de leucotrienos e liberação de histamina ${ }^{5}$. Não há testes in vitro 


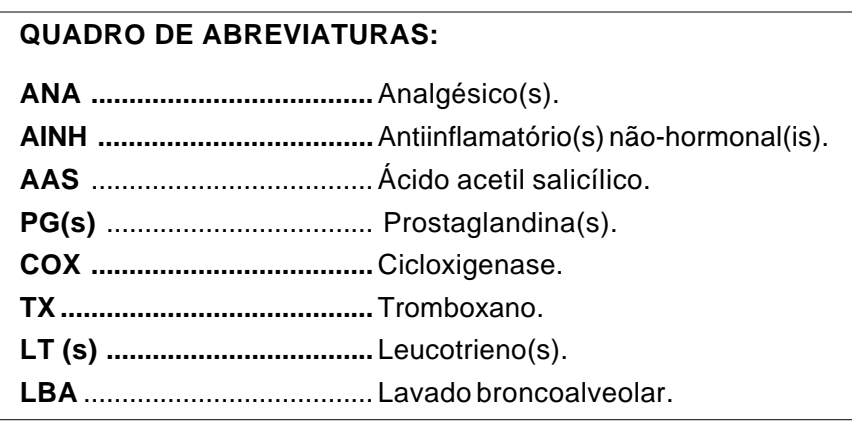

para detecção de sensibilidade ao AAS. O diagnóstico é feito por testes de provocação por via oral ou inalatória ${ }^{5,18-20}$. Os testes por ingestão da droga podem precipitar reações asmáticas ou sistêmicas que necessitem tratamento de urgência. Por isso, devem ser conduzidos em centros especializados e próximos à unidade de terapia intensiva.

O diagnóstico em nossos pacientes não foi confirmado por teste de provocação oral, uma vez que são oriundos de clínica privada e não havia um protocolo para submetê-los com segurança à provocação. No entanto, $58 \%$ dos casos haviam tido reação, em mais de uma ocasião, com a mesma droga ou com medicamentos diferentes, o que aumenta a probabilidade do diagnóstico correto de sensibilidade aos fármacos.

Quiralte et al. ${ }^{10}$ estudaram 240 pacientes com história de intolerância a AINH pela provocação controlada com placebo e único-cega. O teste foi positivo em 98 pacientes, dos quais $74 \%$ eram atópicos e tinham rinoconjuntivite e/ou asma. $\mathrm{Ne}$ nhum deles apresentava pólipo nasal ou urticária crônica. A reação mais freqüentemente observada foi angioedema periorbitário. Outras reações verificadas, mas com menor freqüência, foram erupções cutâneas, asma, reações nasoculares e em quatro casos, reação anafilática. Um aumento significativo de atopia (100\%) foi encontrado em pacientes com angioedema periorbitário isolado. Reatividade cruzada entre os AI NH era comum, mas não foi observada em dois casos com angioedema, um com urticária, um de asma e 18 casos de reação sistêmica ${ }^{10}$.

A freqüência de doenças atópicas associadas à sensibilidade ao AAS depende da população estudada, do tipo de reação e varia entre 10 e 68\% em outras séries. Em crianças, a prevalência de sensibilidade é mais baixa ( 0 a $21 \%$ ) e aumenta após a puberdade 5 . Em nossa casuística, angi oedema foi a manifestação clínica de sensibilidade mais freqüente (86\%). Reação sistêmica foi referida em $30 \%$ e crise de asma em 14\%. Atopia estava presente em $72 \%$ dos casos, freqüência semelhante à observada por Quiral te et al. ${ }^{10}$, especialmente em pacientes com angi oedema periorbitário. As crian- ças responderam clinicamente de modo similar aos adultos. Por outro lado, os asmáticos apresentavam broncoespasmo como manifestação clínica da sensibilidade ao AAS e AINH mais freqüentemente do que aqueles sem asma.

Tipicamente a doença respiratória pelo AAS é adqui rida na idade adulta com raro início na infância ${ }^{6}$. A descrição clássica inclui rinite com pólipo, sinusite, asma e sensibilidade ao AAS. Sem a história de exacerbações associadas ao AAS, é impossível diferenciar asmáticos sensíveis ou não ao AAS ${ }^{9}$. De fato, 2/3 dos pacientes que se enquadram nesta descri ção não reagem ao AAS durante a provocação oral com a droga ${ }^{6}$.

U ma opção para a provocação oral éa utilização do teste nasal com lisina-aspirina para o diagnóstico de asma sensível ao AAS, porém não é empregado em nosso meio. Este difere na dose, duração do período de observação e critérios de positividade ${ }^{18}$. O teste é sensível e específico, mas resultados negativos não excluem a possibilidade de sensibilidade ao AAS. Pode ser usado em pacientes com asma instável e ser o método de escol ha para confirmação de sensibilidade ao AAS somente nos indivíduos com sintomas do trato respiratório superior. Quando negativo, o teste deve ser seguido pela provocação oral ${ }^{18}$.

Sintomas nasais e sinusais são queixas dominantes na mai oria dos pacientes com a tríade do AAS e há evidências de que a rinossinusite agrava a asma $^{15}$. A incidência de sinusite em radiografias de asmáticos sensíveis ao AAS é de até $96 \%$ e a freqüência de pólipos nasais pode alcançar $70 \%{ }^{21}$. Em vários estudos há predomínio do sexo feminino ${ }^{6,10}$.

Em nossa população é alta a freqüência de atopia, e baixa a observação de pólipos nasais, o que indica que são pacientes clinicamente diferentes da anal isada. A mai oria é atópica (72\%), alguns têm polipose nasal (3\%) e alguns desenvolvem broncoespasmo (14\%) à ingestão de AAS/AI NH.

Reações cruzadas entre AAS e paracetamol ocorrem com freqüência baixa que varia entre 0 e $29 \%$ durante provocação com estas drogas ${ }^{22}$. A relação é dependente da dose de paracetamol , e a recomendação é evitar doses mai ores que $1.000 \mathrm{mg}$ desta droga em asmáticos sensíveis ao AAS. Esta freqüência pode ser falseada em razão de se usar doses baixas de paracetamol, pacientes com asma mal controlada, falta de critérios para estabelecer broncoespasmo na espirometria e realização de provocação com paracetamol no período refratário ao $\mathrm{AAS}^{22}$.

Durante os testes de provocação, observou-se que quanto menor a dose de AAS para provocar broncoespasmo maior o número de pacientes que reagem ao paracetamol ${ }^{23}$. Embora nossos casos não tivessem sido submetidos à provocação, pela 
história clínica obtivemos a freqüência de reações ao paracetamol de $11 \%$. Além disso, nenhum paciente reagia isol adamente ao paracetamol, mas já havia tido reação prévia a outro ANA ou AINH.

Paracetamol em doses altas (1.000mg ou maior) deve ser evitado em pacientes sensíveis ao AAS. A maioria dos asmáticos, sensíveis ou não ao AAS, deve tolerar doses de até 650mg com risco pequeno de broncoespasmo ${ }^{23}$. Uma alternativa de tratamento para estes pacientes éa dessensibilização isol ada e cruzada com AAS, reduzindo-se as reações respiratórias pela exposi ção repeti da e crescente ao AAS até que as reações respi ratórias cessem. $O$ estado de dessensibilização permanece por dois a cinco dias com a reatividade retornando após sete dias ${ }^{18}$.

Antagonistas dos leucotrienos e inibidores de sua síntese podem beneficiar estes pacientes, atenuando a broncoconstrição e os sintomas extrapulmonares de pacientes sensíveis ao $\mathrm{AAS}^{23}$. Os anti inflamatórios inibidores sel etivos da COX-2, nimesulida e meloxicam, provocam menos efeitos colaterais gástricos e renais ${ }^{24}$, no entanto faltam estudos que comprovem a segurança do meloxicam em pacientes com sensibilidade a outros AINH.

Pacientes sensíveis a AINH e que toleravam acetaminofen e nimesulida à provocação oral, foram contatados entre um e três anos após o teste para se estabelecer se a tolerância era persistente. Nimesulida era tolerada por 115/122 (94,2\%) e acetaminofen por $71 / 75(95,6 \%)$ dos que haviam ingeri do estas drogas. Somente oito pacientes (5\%) ti veram reações (urticária em sete e crise de asma em um) a uma ou ambas as drogas, mostrando que a provocação identifica os que toleram a droga e que na maioria a tolerância é persistente ${ }^{25}$.

Nimesulida, em 20 asmáti cos sensíveis ao AAS, revelou-se relativamente segura à administração oral, nas doses recomendadas habitualmente e sem efeitos sobre a asma ${ }^{26}$. $\mathrm{E} m$ estudo aberto não controlado, com doses cumulativas de nimesulida, administrada em dois dias diferentes a 429 pacientes com história de sensibilidade a AINH, somente 11 pacientes $(2,6 \%)$ mostraram teste de provocação positivo com nimesulida, revel ando-se adequado em sensibilidade a AINH. No entanto, os autores não mencionam se outros medicamentos foram suspensos antes dos testes e não di scutem o fato de nove destes 11 pacientes terem teste positivo com apenas $10 \mathrm{mg}$ de AAS, o que é incomum ${ }^{27}$.

Os inibidores fracos da COX, benzidamina e salicilato de sódio, raramente desencadeiam sintomas decorrentes da reatividade cruzada com AAS, e estes são dependentes da dose ${ }^{5,28}$. N este grupo de indivíduos com sensibilidade à ANA/AINH verifi- camos que as reações eram freqüentes em atópicos, mais comuns em mulheres adultas, e que as crianças reagiam com as mesmas manifestações clínicas que os adultos. O angioedema palpebral foi a expressão clínica mais freqüente. O broncoespasmo, como era esperado, foi mais comum nos pacientes com asma. A mai oria dos casos havia repetido a reação com mais de uma droga.

\section{SUMMARY}

\section{Clinical findings of sensitivity to analgesics and nonsteroidal antiinflammatory drugs}

BACKGRound. Analgesics (ANA) and nonsteroidal antiinflammatory drugs (NSAID) may simulate an allergic reaction or aggravate asthma and urticaria.

ОвJ ECTIVE. The aim of this study was the analysis of the clinical findings in patients sensitive to ANA/ NSAID.

Methods And subjects. The charts of 183 patients with history of reacting to these drugs were retrospectively analysed.

Results. There were 93 (51\%) females and 90 (49\%) males; 63 (34\%) were aged 15 years or less. Females predominate in the age group older than 15 years $(p=0,02)$. The age by the time of the first reaction varied from 7 months to 65 years (median 15 years). Skin tests to inhalant allergens were positive to at least oneantigen in 100/ 138 patients (72\%).

Clinical presentation was angioedema (86\%); urticaria (39\%); systemic reaction (30\%); nasal and ocular (15\%); and asthma (14\%). The frequency of symptoms was not related to age. Family history of ANA/ NSAID sensitivity was present in $3.8 \%$ of patients. Associated diseases were rhinitis (55\%), chronic urticaria (47\%), asthma (37\%) and conjuntivitis (18\%). Among asthmatics 16 (23,5\%) had had attacks with ANA/NSAID. Nine non asthmatics (7.7\%) had had bronchospasm as clinical presentation. This difference was statistically significant. Repeated reactions occurred in 107 (58\%) patients.

Conclusions. Sensitivity to ANA/ NSAID was frequent in atopics; children and adults responded similarly; reactions were more common in adult females; palpebral angi oedema was the most frequent clinical presentation; bronchosphasm was usually seen in asthmatics and most patients reacted repeatedly to different drugs. [Rev Ass Med Bras; 46(3): 201-6]

KEY WORDS: Asthma. Allergy. Nonsteroidal. Antiinflammatory drugs. Aspirin. 


\section{REFERÊNCIAS BIBLIOGRÁFICAS}

1. Stevenson DD. Adverse reactions to nonsteroidal antiinflammatory drugs. I mmunol Allergy Clin North Am 1998; 18: 773-98.

2. Famaey J P. In vitro and in vivo pharmacological evidence of selective cyclooxygenase - 2 inhibition by nimesulide: An overview. Inflamm Res 1997; 46: 437-46.

3. Vane J R, Botting RM. N ew insights into the mode of action of antiinflammatory drugs. Inflamm Res 1995; 44:1-10.

4. VaneJ R. Themode of action of aspirin and similar compounds. J . Allergy Clin. Immunol 1976; 58:691-712

5. Stevenson DD, Simon RA. Sensitivity to aspirin and nonsteroidal antiinflammatory drugs. In Middleton $E$, Reed CE, Ellis EF, Adkinson NF, Y unginger J W, BusseWW eds. Allergy - Principles and Practice, 5th ed. St. Louis, C.V. Mosby, 1998; p.1225-1234.

6. Stevenson DD. Aspirin-sensitive asthma. In Barnes PJ , Grunstein MM, L eff AR, Wool cock AJ eds. Asthma. Philadel phia, Lippincott-Raven, 1997; p. 2025-2040.

7. Lee TH. Mechanism of bronchospasm in aspirin-sensitive asthma. Am Rev Respir Dis 1993; 148: 1442-3.

8. Mewes $T$, Riechelmann $H$, Klimek $L$. Increased in vitro cysteinyl leukotriene release from blood leukocytes in patients with asthma, nasal polyps, and aspirin intolerance. Allergy 1996; 51: 506-10.

9. Simon RA, Baldwin J L. Drug-induced asthma. In Bierman CW, Pearlman DS, Shapiro GG, Busse WW eds. Allergy, Asthma, and I mmunol ogy from I nfancy to Adulthood. Philadel phia, W.B. Saunders Company, 1998; p. 549-558.

10. Quiralte J , Blanco C, Castillo R, Delgado J, Carrillo T. Intolerance to nonsteroidal antiinflammatory drugs: Results of controlled drug challenges in 98 patients. J Allergy Clin I mmunol 1996; 98: 678-85.

11. Sanchez-Borges M, Capriles-Hullett A. Atopy and NSAID sensitivity. J Allergy Clin Immunol 1997; 100:143-4.

12. Kemp SF, Lockey RF, Wolf BL et al. Anaphylaxis: a review of 266 cases.Arch Intern Med 1995; 155: 1749-54.

13. Almeida MAM, Gaspar AP, Carvalho FS, Nogueira J MA, PintoJ ER. Reacciones adversas a acetaminofen, AAS y AINE en niños: ¿alternativas? Allergy Asthma Proc. 1998; 12:30-5.

14. Feigenbaum BA, Simon RA, Stevenson DD. Aspirin intolerance. Ann Allergy 1994; 73: 455-6.
15. Kowalski ML. Aspirin-sensitive rhinosinusitis/asthma syndrome - pathophysiology and management. Allergy Clin Immunol 1996; 8:49-56.

16. Ferreri NR, Howland WC, Stevenson DD et al. Release of leukotrienes, prostaglandins, and histamine into nasal secretions of aspirin-sensitive asthmatics during reaction to aspirin. Am Rev Respir Dis 1988; 137: 847-854

17. SladekK K, Dworski R, Soja J et al. Eicosanoids in bronchoalveolar lavage fluid or aspirin intolerant patients with asthma after aspirin challenge. Am J Respir Crit Care Med. 1994; 149: 940 - 946

18. Milewski M, Mastalerz L, Nizankowska E, Szczeklik A. Nasal provocation test with lysine-aspirin for diagnosis of aspirinsensitive asthma. J Allergy Clin I mmunol 1998; 101: 581-6

19. Stevenson D.D. Challenge procedures in detection of reactions to aspirin and nonsteroidal anti-inflammatory drugs. Ann Allergy 1993; 71: 417-8.

20. TomazEM; Ferreira MF; Spinola MA, Oliveira ML, ClodeMH, Palma-Carlos AG. Pruebas deprovocación nasal en el diagnostico de la urticaria inducida por ácido acetilsalicílico. Allergy Asthma Proc 1998; 12:36-40.

21. Settipane GA. Nasal polyps: E pidemiology, pathology, immunology and treatment. Am J Rhinol 1987; 1: 119 - 126

22. Settipane RA et al. Prevalence of cross-sensitivity with acetaminophen in aspirin-sensitive asthmatic subjects. J Allergy Clin I mmunol 1995; 96: 480-5.

23. Hendersen WR. Therole of leukotrienes in inflammation. Ann Intern Med 1994; 121: 689 - 697.

24. Brusasco V, Crimi E, Scaricabarozzi I. Nimesulide does not interfere with airway responsiveness in allergic asthma. Drugs 1993; 46 (suppl 1): 121-3

25. Quarantino D, Romano A, Papa G et al. Long-term tolerability of nimesulide and acetaminophen in nonsteroidal antiinflammatory drug-intolerant patients. Ann Allergy, Asthma \& Immunol 1997; 79: 47-50.

26. Bianco S, Robuschi M, Petrigni G et al. Efficacy and tolerability of nimesulide in asthmatic patients intolerant to aspirin. Drugs 1993; 46 (suppl 1): 115-20.

27. Andri L, Senna G, Betteli $C$ et al. Tolerability of nimesulide in aspirin-sensitive patients. Ann Allergy 1994; 72: 29-32.

28. Vane J R, Botting RM. Mechanism of action of antiinflammatory drugs. Scand J Reumatol 1996; 25 (suppl 102): 9-21. 